\title{
Taenia ovis: an emerging threat to the Chinese sheep industry?
}

\author{
Yadong Zheng ${ }^{1,2}$
}

\begin{abstract}
Background: Taenia ovis is a tapeworm that is mainly transmitted between dogs and sheep. Although T. ovis infection is not a public health issue, it causes a great financial loss due to condemnation of carcasses. The first outbreak of $T$. ovis infection in China occurred in 2015. Reassessment of adverse effects of $T$. ovis infection on Chinese sheep industry in future is necessary.

Discussion: The first $T$. ovis outbreak in China suggests that the epidemic situation across the country is underestimated. For the transmission of T. ovis, many factors, including eggs, dogs and wild canids, human behaviours and sheep trade, should be seriously considered. In blocking the transmission chain, regular treatments of the infected dogs using anthelmintics play a crucial step, but at the moment it is difficult to be fully executed in China, largely due to the behaviours, customs and faith of local farmers. Moreover, combined with no clinical symptoms in the infected adult sheep and goats, the lack of pre-mortem diagnostic tools makes it harder to practice a national wide surveillance as well as inspection and quarantine in increasingly frequent free sheep trade activities in China, leading to an inability to restrict $T$. ovis infection into small areas. Furthermore, the ongoing campaigns against Echinococcus granulosus may have an adverse effect on control of $T$. ovis infection because of no consideration of a role of dogs in the transmission of the parasite.

Conclusion: Lack of national epidemic data, pre-mortem diagnostic reagents and vaccines severely hampers the implementation of disease control campaigns and the restriction of $T$. ovis infection into small areas. Consequently, sheep and goats are at an increasing risk of T. ovis exposure and the possibility of large-scale outbreaks across China in future is possible, causing great adversity towards sheep industry.
\end{abstract}

Keyword: Taenia ovis, Sheep, Goat, Sheep industry

\section{Background}

Taenia ovis is a tapeworm that resides in the small intestine of dogs. With dog faeces, $T$. ovis eggs are released into the surrounding environment and develop into larvae predominately in body muscles, but occasionally in the liver, lung, kidney and brain, when sheep and goats ingest egg-contaminated water or forage. After nearly 1.5 months of development in the muscles, these larval parasites become infective [1], and dogs are infected with the ingestion of contaminated muscles or organs, thus completing a life cycle.

\footnotetext{
Correspondence: zhengyadong@caas.cn

${ }^{1}$ State Key Laboratory of Veterinary Etiological Biology, Key Laboratory of Veterinary Parasitology of Gansu Province, Lanzhou Veterinary Research Institute, CAAS, 1 Xujiaping, Yanchangbu, Lanzhou 730046, Gansu, China ${ }^{2}$ Jiangsu Co-innovation Center for Prevention and Control of Important Animal Infectious Diseases and Zoonoses, Yangzhou 225009, China
}

Taenia ovis is distributed across the world and its outbreaks have had a tendency to be sporadic in the past decade [2-5]. In 2015, a T. ovis outbreak was first reported in Gansu Province in the northwestern part of China, and more than one half of sheep in a farm were infected, with an economic loss of up to $\$ 16,000$ [6]. Therefore, the reassessment of adverse effects of $T$. ovis infection on the Chinese sheep industry in future is necessary. Although T. ovis infection is not a public health issue, it hampers the development of the sheep industry by having an adverse effect on sheep trade and causing a great financial loss due to condemnations of carcasses $[4,7]$. Considering multiple factors including the epidemiological features, lack of diagnostic tools and vaccines, a huge quantity of sheep and goats (> 146 million in 2007 in China) and traditional breeding management, sheep and goats are at a high risk of $T$. ovis exposure and it is potential to deteriorate the 
Chinese sheep industry in future due to the possibility of a wide spread of $T$. ovis infection.

\section{Discussion}

Although the first outbreak does not rule out the possibility of imported cases, it may have resulted from the spread of T. ovis present in remote areas or regions in China [6], and also suggest that the epidemic situation across the country is underestimated. The assessment of the epidemiology of $T$. ovis is urgent because the lack of epidemic data makes it difficult and inefficient to implement disease control campaigns, such as an ongoing programme called Ovis Management Limited in New Zealand [7]. For the transmission of T. ovis, many factors, including eggs, dogs and wild canids, human behaviours and sheep trade, should be carefully considered. It was estimated that one adult worm could produce 70,000 250,000 eggs per day [7], posing a huge resource for pasture and water contamination. Although the developmental and maturation processes of eggs in the environment are largely unknown, they maintain infectivity for as long as one year on pasture [8]. The movement of sheep, wind, birds and rainwater may contribute to the dissemination of eggs from one place to another [7], which makes more flocks of sheep and goats at a risk of egg exposure. Therefore, effective control of the infected dogs, an original source of $T$. ovis eggs, plays a crucial step in blocking the transmission chain. The control measures include regular treatments of dogs with anthelmintics and a ban on feeding dogs with contaminated muscles or organs. It seems impossible to fully implement these measures in remote rural areas, especially in Gansu Province, Qinghai Province and Xinjiang Autonomous Region where people usually practice home-slaughter, and owners feed dogs with offal and are not willing to treat dogs with anthelmintics due to their customs and faith. These human behaviours are also considered to contribute, to a large degree, to the current high prevalence of cystic echinococcosis in China, caused by a small tapeworm, Echinococcus granulosus, that is also transmitted mainly between sheep and dogs [9]. Moreover, the role of wild canids in the spread of $T$. ovis remains elusive. Although T. ovis infection was reported in wolves, it is still controversial that wolves can act as definitive hosts $[7,10]$. Coyotes have been suggested to transmit $T$. ovis [7], but it is still not yet verified. If wild canids, including wolves and foxes, are really involved in the transmission of $T$. ovis as definitive or reservoir hosts, the epidemic status becomes more complicated because of the full implementation of The Ecologic Restoration Plan in recent years in China, which favours returning farmland to forestry. Furthermore, increasing free animal trade activities in China would also accelerate the rapid spread of $T$. ovis from one place to another, making outbreaks unpredictable. Under these circumstances, the variables involved in transmission significantly increase the risk of exposure to $T$. ovis for both sheep and goats.

Except for Taenia krabbei, which is morphologically indistinguishable from $T$. ovis and is transmitted between carnivores and cervids [11], T. ovis is easily identified based on the morphological traits, host-preference and organotropism. Unfortunately, approaches for pre-mortem diagnosis of $T$. ovis infection in sheep are unavailable. At present, meat inspection is commonly used for identification of T. ovis infection. It should be noted that, as the sensitivity of meat inspection heavily relies on the intensity of infection, the true prevalence of T. ovis will be remarkably underestimated by utilization of abattoir data [7]. Taenia ovis infection in adult sheep and goats generally presents no clinical manifestations, which causes it to always be neglected. Combined with no clinical symptoms in the infected adults, the lack of pre-mortem diagnostic tools makes it harder to practice a national wide surveillance as well as inspection and quarantine in frequent free sheep trading in China. Therefore, the development of diagnostic reagents for pre-mortem detection of $T$. ovis infection, such as serological tests like enzyme-linked immunosorbent assay (ELISA), is urgently required.

One of the optimal control strategies is to vaccinate animals. However, although several antigens have been found to induce high protection against $T$. ovis infection even in field trials, no vaccines are commercially available up to now [12]. Treatment of infected sheep and goats using anthelmintics is likely unnecessary because $T$. ovis metacestode-contaminated carcasses are still subjected to condemnation rather than for human consumption. For the elimination of egg contamination, effective anthelmintics including praziquantel are available for the treatment of dogs, which is one of the core strategies of The Hydatid Control Plan (HCP) that is now being fully implemented in the echinococcosis-epidemic areas in China. It should be pointed out that the HCP's implementation may have an adverse effect on eradication of $T$. ovis because owners are only educated not to feed dogs with offal including liver and lung, so they may feed dogs with cyst-containing carcasses. A lesson may be learnt from New Zealand, where the infectious rate of $T$. ovis was rather low prior to 1950 but increased substantially in the 1960's, largely due to the implementation of anti-E. granulosus campaigns [7]. Therefore, it is highly recommended to make modifications of the on-going HCP strategies with full consideration of T. ovis transmission.

\section{Conclusion}

Mainly due to lack of national epidemic data and premortem diagnostic reagents, it is very difficult to efficiently implement disease control campaigns and restrict $T$. ovis infection to small areas. Consequently, the exposure of 
sheep and goats to T. ovis is notably increased and the possibility of large-scale outbreaks across China in future is possible, causing considerable problems for the sheep industry.

\title{
Abbreviations
}

ELISA, enzyme-linked immunosorbent assay; HCP, Hydatid Control Plan

\section{Acknowledgements}

The author would like to thank the reviewer and editor for their constructive suggestions.

\section{Funding}

The study was financially supported by National Natural Science Foundation of China (No. 31472185 and 31201900) and National Key Basic Research Program (973 program) of China (No. 2015CB150300).

\section{Availability of data and material}

\author{
Not applicable.
}

\section{Competing interests}

The author declares that he has no competing interests.

\section{Consent for publication}

Not applicable.

\section{Ethics approval and consent to participate} Not applicable.

Received: 20 June 2016 Accepted: 14 July 2016

Published online: 26 July 2016

\section{References}

1. DeWolf BD, Poljak Z, Peregrine AS, Jones-Bitton A, Jansen JT, Menzies PI. Development of a Taenia ovis transmission model and an assessment of control strategies. Vet Parasitol. 2013:198:127-35.

2. Eichenberger RM, Karvountzis S, Ziadinov I, Deplazes P. Severe Taenia ovis outbreak in a sheep flock in south-west England. Vet Rec. 2011;168:619.

3. Sissay MM, Uggla A, Waller PJ. Prevalence and seasonal incidence of larval and adult cestode infections of sheep and goats in eastern Ethiopia. Trop Anim Health Prod. 2008:40:387-94.

4. DeWolf BD, Peregrine AS, Jones-Bitton A, Jansen JT, Mactavish J, Menzies PI. Distribution of, and risk factors associated with, sheep carcass condemnations due to Cysticercus ovis infection on Canadian sheep farms. Vet Parasitol. 2012;190:434-41.

5. Oryan A, Goorgipour S, Moazeni M, Shirian S. Abattoir prevalence, organ distribution, public health and economic importance of major metacestodes in sheep, goats and cattle in Fars, southern Iran. Trop Biomed. 2012;29:349-59.

6. Shi W, He W, Guo X, Liu Q, Gao S, Zhan F, et al. The first outbreak of Taenia ovis infection in China. Parasitol Int. 2016:65:422-3.

7. De Wolf BD, Peregrine AS, Jones-Bitton A, Jansen JT, Menzies PI. Taenia ovis infection and its control: a Canadian perspective. N Z Vet J. 2014;62:1-7.

8. Lawson JR, Gemmell MA. Hydatidosis and cysticercosis: the dynamics of transmission. Adv Parasitol. 1983;22:261-308.

9. Craig PS, McManus DP, Lightowlers MW, Chabalgoity JA, Garcia HH, Gavidia CM, et al. Prevention and control of cystic echinococcosis. Lancet Infect Dis. 2007;7:385-94

10. Moks E, Jogisalu I, Saarma U, Talvik H, Jarvis T, Valdmann H. Helminthologic survey of the wolf (Canis lupus) in Estonia, with an emphasis on Echinococcus granulosus. J Wildl Dis. 2006;42:359-65.

11. Al-Sabi MN, Chriel M, Holm E, Jensen TK, Stahl M, Enemark HL. Reappearance of Taenia ovis krabbei muscle cysts in a roe deer (Capreolus capreolus) in Denmark after 60+ years. Vet Parasitol. 2013;196:225-9.

12. Lightowlers MW. Cestode vaccines: origins, current status and future prospects. Parasitology. 2006;133(Suppl):S27-42.

\footnotetext{
Submit your next manuscript to BioMed Central and we will help you at every step:

- We accept pre-submission inquiries

- Our selector tool helps you to find the most relevant journal

- We provide round the clock customer support

- Convenient online submission

- Thorough peer review

- Inclusion in PubMed and all major indexing services

- Maximum visibility for your research 